\title{
Family Centered Music Therapy for Preterm Infants and Their Parents in the NICU: A Mixed-Method Study
}

\author{
Yakobson $D^{1,2}$, Elefant $C^{3}$, Litmanovitz $\mathrm{I}^{1,4}$, Bauers $\mathbf{S}^{1,4}$, \\ Arnon S ${ }^{1,4}$ \\ ' Neonatal Department, Meir Medical Center, Kfar Saba, Israel \\ ${ }^{2}$ Doctoral Program in Music Therapy, Institute for Communication and \\ Psychology, Aalborg University, Denmark \\ ${ }^{3}$ School for Creative Arts Therapies, University of Haifa, Israel \\ ${ }^{4}$ Sackler Faculty of Medicine, Tel Aviv University, Tel Aviv, Israel
}

\section{Background}

Preterm infants experience noxious stress during their NICU hospitalization. Unbuffered stress may negatively influence their autonomic nervous system (ANS) maturation thus effecting neurobehavior and development. ${ }^{1}$ Music therapy (MT) is an evidence-based intervention used in neonatal settings. ${ }^{2}$ When coupled with skin-to-skin care (SSC) it may reduce stress responses in both preterm infants and their parents and enhance family-centered care. ${ }^{3,4}$ Few studies have investigated these interventions combined, and evidence of longer-term effects is missing.

\section{Aim}

To investigate the effects of combined, family-centered MT and SSC on preterm-infants' ANS stability and their parents' mental well-being.

\section{Methods}

An embedded mixed-methods design was used. ${ }^{5}$ A single-center, cluster-randomized trial with two parallel arms was conducted. Ten time-clusters of two months, each with five - eight participating families, were randomized to either combined MT+SSC or SSC alone. Sixty-eight families were offered two weekly sessions of the allocated condition in the NICU, and a follow up at home at three-months corrected age (CA). The primary outcome was stabilization of the ANS as defined by change in the high frequency component of heart rate variability during the second session. Secondary outcomes included additional HRV measures, attachment, and parental anxiety. Outcomes were analyzed on an intention-to-treat basis using linear mixed-effects models.

A qualitative exploration of parents' experiences regarding MT sessions was performed. Ten parents participated in semi-structured interviews at four-months CA. Data was analyzed using thematic analysis methodology within a reflexive hermeneutic approach.

The MT intervention was based on the "First sounds: Rhythm, Breath and Lullaby" model ${ }^{6}$, which focuses on the parent-infant dyad. Key elements include specific attention to infant's cues, parent, and environment, and to their reciprocal interaction; rhythmic entrainment; use of parents' voice and musical heritage; and encapsulating the sounds of the intrauterine environment. Guidelines for the intervention afforded high flexibility to address alternating parental or infants' needs, and to offer additional means for musical engagement.

\section{Results/Finding}

MT+SSC improved infants' ANS stability, as indicated by a greater increase in HF power (mean difference $5.19 \mathrm{~m} 2 / \mathrm{Hz}$, $\mathrm{SE}=1.27, \mathrm{p}<0.001)(95 \%$ confidence interval 0.87 to 2.05$)$, as compared to SSC alone. HF power is an indicator of a relaxed state in the ANS, representing improvement in autonomic regulation, which highly impacts preterm-infants' processes of recovery and maturation. ${ }^{7}$

Other HRV indices as well as parental anxiety showed a nonsignificant tendency favoring MT+SSC condition. Main themes derived from parents' interviews revealed that $\mathrm{MT}+\mathrm{SSC}$ sessions were perceived as unique moments solely dedicated to connecting with their baby, and as such, essentially different from daily medical routines. Singing was experienced as means to provide "something deep from within themselves" and enhanced feelings of self-coping by overcoming personal difficulties. Parents reported that MT has promoted relaxation for both them and their baby, leading to feelings of intimacy and being-in-the moment. MT informed and motivated their use of music throughout the hospitalization period and was incorporated into their everyday lives.

\section{Relevance to NIDCAP}

This study relates to core components of the NIDCAP model, namely, modulation of stress in the NICU, promotion of infant's behavioral organization, and enhancement of early parent- infant bonding. ${ }^{8}$ Implementation of family centered MT during SSC might provide additional opportunities to enhance stability, intimacy, and bonding in the NICU.

\section{Conclusions}

Combined MT+SSC contributes to preterm-infants' ANS stability and stress reduction, laying an important foundation for neuro-behavioral and psycho-emotional development. Family- centered MT is highly attuned to parents' and infants' needs and may support the dyad to engage musically and enhance meaningful interactional moments during hospitalization. Studies evaluating longer-term effects of MT on preterm infants' development are warranted, as well as the investigation of longer therapeutic processes and their impact on parents' experiences. 